\title{
Capsule endoscopy with retention of the capsule in a duodenal diverticulum
}

The most important complication of capsule endoscopy is retention of the capsule. Among patients with obscure gastrointestinal bleeding, capsule retention was reported in $1.5 \%$; in patients with suspected Crohn's disease or suspected stenosis of the small bowel the risk of capsule retention seems to be considerably higher (5\% and $21 \%$ respectively) $[1,2]$. To our best knowledge this is the first case of capsule retention in a duodenal diverticulum.

A 74-year-old woman was admitted to hospital for evaluation of microcytic anemia. Gastroscopy and colonoscopy were normal. Capsule endoscopy, using the M2A capsule (Given Imaging Ltd., Yoqneam, Israel [3]), demonstrated some diverticula shortly after passage of the pylorus. Further images were not evaluable. Three weeks afterwards the patient was asymptomatic but had still not excreted the capsule. A plain film of the abdomen demonstrated the capsule superimposed on the epigastrium and was otherwise normal ( Fig.1). A small-bowel radiograph with water-soluble contrast medium showed the capsule in a duodenal diverticulum and ruled out obstruction of the small bowel ( Fig.2). Gastroscopy was performed and the capsule successfully extracted from a large juxtapapillary diverticulum using the Roth retrieval net (๑ Fig. 3).

Most patients with capsule retention are asymptomatic. However, a very few cases of symptomatic bowel obstruction requiring surgical or endoscopic removal of the impacted capsule have been reported $[4,5]$. This is the first case of capsule retention in a duodenal diverticulum and successful endoscopic removal of the impacted capsule. We suggest that, even in asymptomatic patients, capsules that are retained in intestinal diverticula and are not excreted within a period of about 3 weeks should be removed by gastroscopy or enteroscopy in order to prevent complications such as diverticulitis, perforation, or pancreatitis.

We conclude that capsule retention in a duodenal diverticulum is a rare complica-

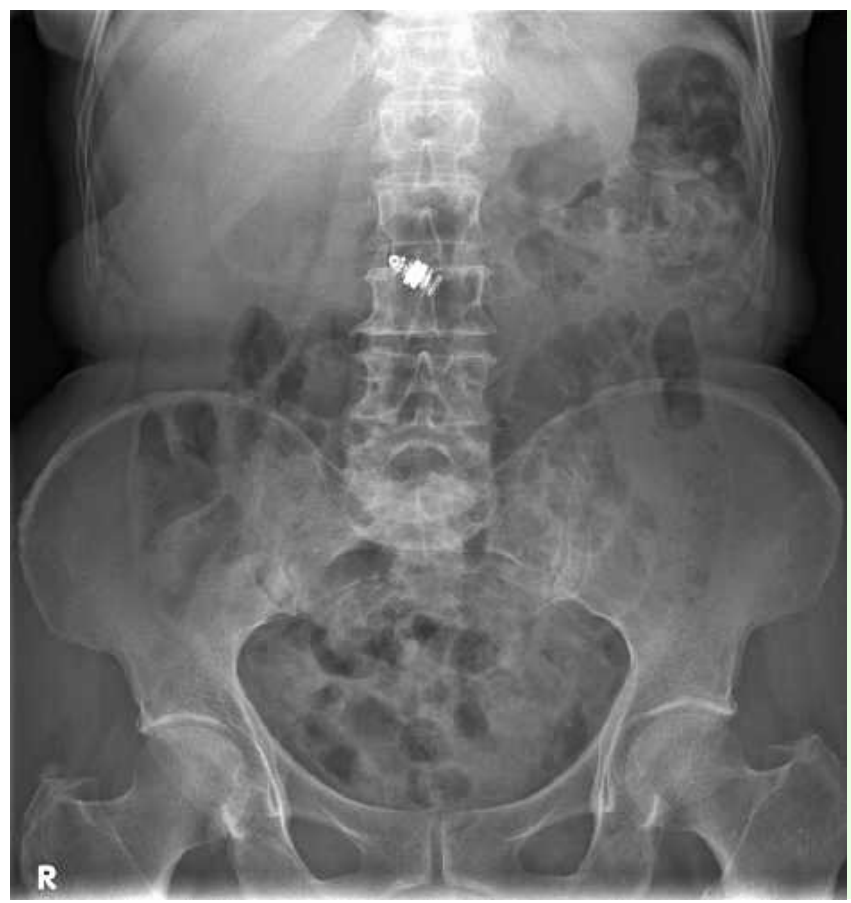

Fig. 1 Plain film anteroposterior abdominal radiograph: the capsule is superimposed on the epigastrium.

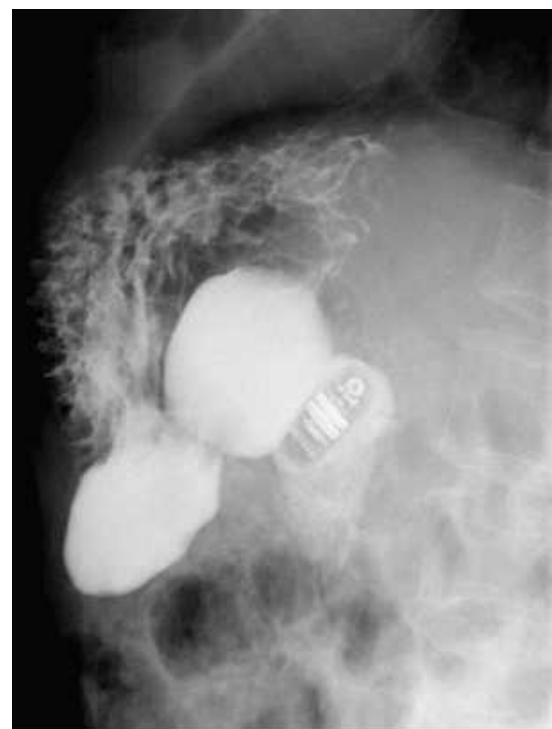

Fig. 2 Small-bowel radiograph with watersoluble contrast medium: the capsule is retained in a large duodenal diverticulum.

tion of capsule endoscopy. In our patient endoscopic removal of the impacted capsule using a retrieval net was successful.

Endoscopy_UCTN_Code_CPL_1AI_2AB

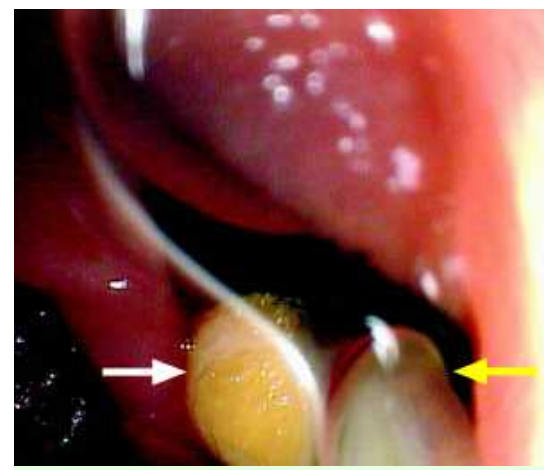

Fig. 3 The capsule was captured in a Roth retrieval net (white arrow). The yellow arrow marks the Teflon-coated tube of the net. 
P. Ordubadi ${ }^{1}$, B. Blaha ${ }^{1}$, A. Schmid ${ }^{2}$,

W. Krampla ${ }^{3}$, W. Hinterberger ${ }^{2}$,

M. Gschwantler ${ }^{1}$

1 Fourth Department of Internal Medicine, Wilhelminenspital, Vienna, Austria

2 Second Department of Internal Medicine, Donauspital, Vienna, Austria

3 Department of Radiology, Donauspital, Vienna, Austria

\section{References}

1 Lewis $B$. How to prevent endoscopic capsule retention. Endoscopy 2005; 37: 852 - 856

2 Lewis B. Capsule endoscopy - transit abnormalities. Gastrointest Endosc Clin N Am 2006; 16: $221-228$

3 Mata A, Llach J, Bordas JM. Wireless capsule endoscopy. World J Gastroenterol 2008; 13 : 1969-1971

4 Sears DM, Avots-Avotins A, Culp K, Gavin $M W$. Frequency and clinical outcome of capsule retention during capsule endoscopy for GI bleeding of obscure origin. Gastrointest Endosc 2004; 60: 822 - 827

5 Hara AK, Leighton JA, Sharma VK, Fleischer $D E$. Small bowel: preliminary comparison of capsule endoscopy with barium study and CT. Radiology 2004; 230: 260-265
Bibliography

DOI $10.1055 / \mathrm{s}-2008-1077563$

Endoscopy 2008; 40: E247-E248

(c) Georg Thieme Verlag KG Stuttgart · New York . ISSN 0013-726X

Corresponding author

M. Gschwantler, MD

Wilhelminenspital

4th Department of Internal Medicine

Montleartstraße 37

1160 Vienna

Austria

Fax: +43-1-491502409

Michael.Gschwantler@wienkav.at 